\title{
Impaired Global Myocardial Flow Dynamics Despite Normal Left Ventricular Function and Regional Perfusion in Chronic Kidney Disease: A Quantitative Analysis of Clinical ${ }^{82} \mathbf{R b}$ PET/CT Studies
}

\author{
Kenji Fukushima ${ }^{1}$, Mehrbod S. Javadi ${ }^{1}$, Takahiro Higuchi ${ }^{1}$, Paco E. Bravo ${ }^{1}$, David Chien ${ }^{1}$, Riikka Lautamäki ${ }^{1}$, \\ Jennifer Merrill ${ }^{1}$, Stephan G. Nekolla ${ }^{2}$, and Frank M. Bengel ${ }^{1,3}$ \\ ${ }^{1}$ Division of Nuclear Medicine, Russell H. Morgan Department of Radiology, Johns Hopkins University, Baltimore, Maryland; \\ ${ }^{2}$ Nuklearmedizinische Klinik der Technischen Universität München, Munich, Germany; and ${ }^{3}$ Klinik für Nuklearmedizin, Medizinische \\ Hochschule Hannover, Hannover, Germany
}

\begin{abstract}
Impaired global myocardial flow reserve (MFR) may be associated with increased risk for cardiac events and coronary artery disease progression. Chronic kidney disease (CKD) is also considered a risk factor for cardiovascular disease. We sought to investigate the effect of CKD on the myocardial microcirculation in patients referred for clinical ${ }^{82} \mathrm{Rb} \mathrm{PET} / \mathrm{CT}$, who had normal left ventricular (LV) function and no flow-limiting coronary artery disease. Methods: Estimated glomerular filtration rate (eGFR) was available for 230 patients who had undergone rest and pharmacologic stress ${ }^{82} \mathrm{Rb}$ PET/CT for suspected coronary artery disease. CKD was defined as an eGFR less than 60 $\mathrm{mL} / \mathrm{min} / 1.73 \mathrm{~m}^{2}$. After patients with hemodialysis, a renal transplant, abnormal regional perfusion (summed stress score $>4$ ), or reduced LV function (LV ejection fraction $<45 \%$ ) were excluded, 40 CKD patients remained. Those were compared with a control group without CKD, which was matched for age, sex, coronary risk factors, and systemic hemodynamics $(n=42)$. List-mode acquisition of PET enabled quantification of myocardial blood flow (MBF) and MFR using a previously validated retention model with correction for ${ }^{82} \mathrm{Rb}$ extraction. Rest MBF was normalized to rate-pressure product. Results: Mean eGFR in the CKD group was reduced ( $44 \pm 14$ vs. $99 \pm 28 \mathrm{~mL} / \mathrm{min}$ / $1.73 \mathrm{~m}^{2} ; P<0.0001$ ), and creatinine was significantly elevated, compared with controls $(1.9 \pm 1.1$ vs. $0.8 \pm 0.2 \mathrm{mg} / \mathrm{dL} ; P<$ $0.0001)$. MFR was significantly reduced in CKD $(2.2 \pm 1.0$ vs. $3.0 \pm 1.2$ for controls; $P=0.027)$. This reduction was mainly due to increased rest MBF $(1.1 \pm 0.4$ in CKD vs. $0.8 \pm 0.2 \mathrm{~mL}$ $\mathrm{min} / \mathrm{g}$ in controls; $P=0.007)$. Stress myocardial flow was comparable between both groups $(2.3 \pm 0.9$ vs. $2.3 \pm 0.8 \mathrm{~mL} / \mathrm{min} / \mathrm{g}$; $P=0.08)$. Overall, MFR was significantly correlated with eGFR $(r=0.41 ; P=0.0005)$. Stress MBF did not correlate with eGFR $(r=0.002 ; P=0.45)$, but rest MBF showed an inverse correlation $(r=-0.49 ; P<0.0001)$. Rest MBF was also inversely correlated with hemoglobin $(r=-0.28 ; P=0.014)$, but only eGFR was an independent correlate at multivariate analysis.
\end{abstract}

\footnotetext{
Received Oct. 12, 2011; revision accepted Feb. 6, 2012.

For correspondence or reprints contact: Frank M. Bengel, Department of Nuclear Medicine, Hannover Medical School, Carl-Neuberg-Strasse 1, D-30625 Hannover, Germany.

E-mail: Bengel.Frank@mh-hannover.de

Published online May 4, 2012.

COPYRIGHT @ 2012 by the Society of Nuclear Medicine, Inc.
}

Conclusion: MFR is impaired in patients with renal insufficiency with normal regional perfusion and LV function, mostly because of elevated rest flow. Absolute quantification of flow may be useful to identify microvascular dysfunction as a precursor of clinically overt coronary disease in this specific risk group.

Key Words: positron emission tomography; myocardial blood flow; renal dysfunction; chronic kidney disease; coronary artery disease

J Nucl Med 2012; 53:887-893

DOI: 10.2967/jnumed.111.099325

C hronic kidney disease (CKD) is known to be a major risk factor for coronary artery disease (CAD). CKD is closely linked to adverse cardiac events and mortality, and approximately $50 \%$ of patients with renal insufficiency may present with cardiac dysfunction. CKD is found in more than $30 \%$ of subjects with acute heart failure onset (1). This linkage has become widely recognized as the socalled cardiorenal syndrome $(1,2)$. Sudden cardiac death, myocardial infarction, and heart failure are frequently found in patients with end-stage renal disease (3). More notably, however, it has also been reported that $\mathrm{CKD}$ is associated with unfavorable cardiac outcome in earlier stages of kidney disease and in the absence of obstructive CAD (4). Microvascular dysfunction may be present under such circumstances, and it may contribute to a more rapid progression toward overt CAD (5).

Currently, radionuclide myocardial perfusion imaging is widely used to detect CAD in the clinical setting. SPECT is most widely used for this purpose, and its value has been proven in renal insufficiency $(1,2)$. But SPECT is limited to a relative regional analysis of myocardial perfusion, for detection of ischemia or infarction in a respective vascular territory. Global microvascular dysfunction, as a potential precursor of these conditions, cannot be identified. PET, on the other hand, has increasingly penetrated the clinical 
arena. PET may not only offer improved accuracy for detection of relative regional ischemia (6) but also may be used, with several agents-including the clinically most frequently used tracer, ${ }^{82} \mathrm{Rb}$ - for quantitative measurement of global myocardial blood flow (MBF) and myocardial flow reserve (MFR) as additional diagnostic and prognostic markers $(7,8)$.

The aim of our study was to determine the presence of impaired MFR in patients with CKD and clarify the severity of coronary endothelial dysfunction.

\section{MATERIALS AND METHODS}

\section{Patient Enrollment}

Two hundred thirty consecutive patients (mean age \pm SD, $58 \pm$ 12 y; men, 99; women, 131), who were referred for clinical rest or dipyridamole myocardial perfusion PET with available estimated glomerular filtration rate (eGFR) values $\left(\mathrm{mL} / \mathrm{min} / 1.73 \mathrm{~m}^{2}\right.$, according to the Modification of Diet in Renal Disease) (9), were included in this analysis. Patients on hemodialysis or those with a renal transplant were excluded. Other exclusion criteria were known myocardial disease, cardiomyopathy, acute or old myocardial infarction, unstable angina, history of heart failure, and coronary revascularization. Patients with abnormal results of clinical PET perfusion imaging - that is, those with relative regional perfusion defects (summed stress score, $>4$ ) or reduced cardiac function (left ventricular [LV] ejection fraction, $<45 \%$ )-were also excluded. Among the remaining 166 patients, 40 had an eGFR below 60. Those were assigned to the CKD group (Fig. 1). From the remaining patients with an eGFR over 60, a control group of 42 subjects was selected by individual matching with the

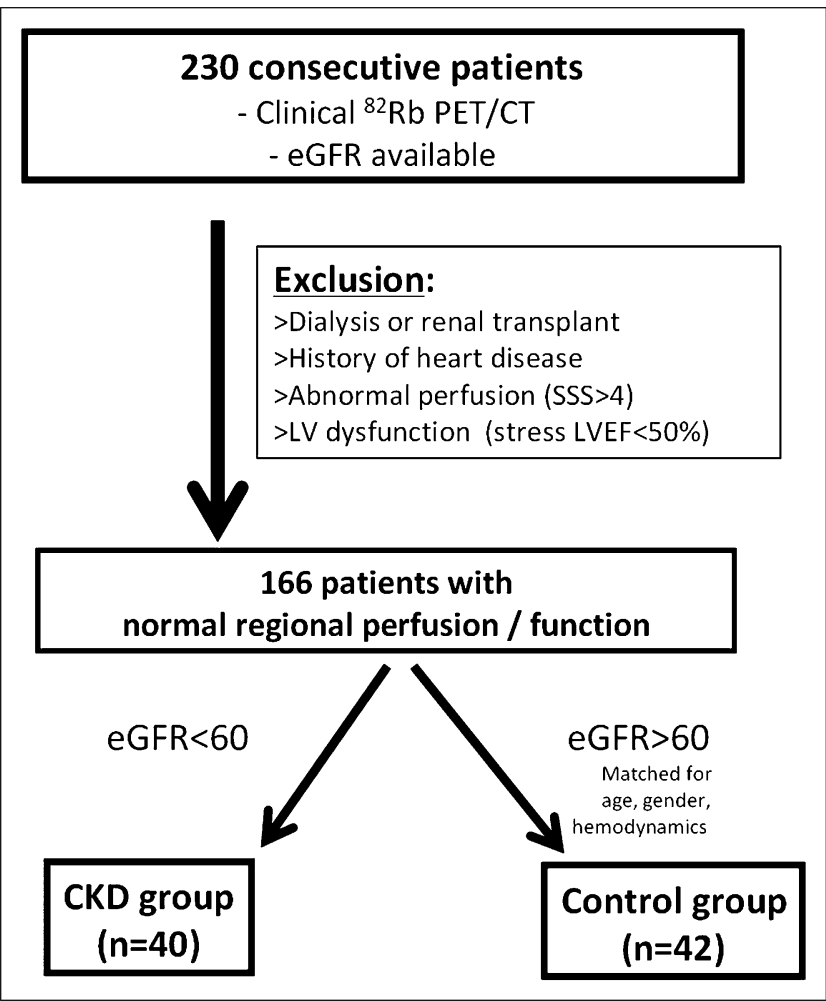

FIGURE 1. Flow chart showing patient selection.
CKD patients. Matching criteria included the clinical profile (age, sex, nonrenal risk factors, and medication) and hemodynamics (baseline heart rate, blood pressure, and ejection fraction). Group characteristics are shown in Table 1. A hypertensive patient was defined as one who had a systolic blood pressure above 140/90 or was taking antihypertensive medication. A diabetic patient was defined as one who had a blood glucose level above $120 \mathrm{mg} / \mathrm{dL}$ or was taking antidiabetic medication. A dyslipidemic patient was defined as one who had an increased level of total cholesterol, lowdensity lipoprotein, and triglyceride or was taking lipid-lowering medication. Hemodynamic profiles were collected from recorded data during rest or stress PET.

This retrospective analysis was granted exempt status by the Johns Hopkins Institutional Review Board (protocol NA_ 00006886).

\section{PET Acquisition Protocol}

A 64-slice Discovery Rx VCT PET/CT scanner (GE Healthcare) was used. Patients fasted for more than $4 \mathrm{~h}$ and were instructed to refrain from caffeinated substances or theophyllinecontaining medication for $24 \mathrm{~h}$ before testing. After giving informed consent, individuals were positioned, and a low-dose CT scan (120 kV, 50-100 mA) for attenuation correction of PET data was acquired during shallow breathing. ${ }^{82} \mathrm{Rb}$-chloride $(1,480$ $1,850 \mathrm{MBq}$ [40-50 $\mathrm{mCi}]$ ) was infused using a large antecubital intravenous line, and list-mode 2-dimensional PET images were acquired for $8 \mathrm{~min}$. Then, infusion of dipyridamole was started $(0.56 \mathrm{mg} / \mathrm{kg}, 4 \mathrm{~min})$, and a second dose of ${ }^{82} \mathrm{Rb}$-chloride $(1,480$ $1,850 \mathrm{MBq}$ [40-50 $\mathrm{mCi}]$ ) was infused $4 \mathrm{~min}$ after completion, followed by an 8-min list-mode acquisition. Quality control of PET and CT for attenuation correction was performed, and datasets were realigned if necessary (10). List-mode data were resampled to attenuation-corrected static ( 90 -s prescan delay), electrocardiographically gated ( 8 bins), and dynamic images (32 frames; $20 \times 6,5 \times 12,4 \times 30$, and $3 \times 60 \mathrm{~s}$ ).

\section{PET Analysis and Flow Measurement}

Relative Regional Perfusion. Static images were reangulated to create short- and long-axis slices for visual analysis of regional perfusion. Each of 17 myocardial segments (11) was scored on a scale of 0-4 (normal, mildly decreased, moderately decreased, severely decreased, or absent perfusion) by experienced readers who were masked to other data. The summed stress score was calculated (12), and abnormal perfusion was defined as a summed stress score over 4.

LV Function. Endocardial contours were identified on gated series to determine LV volumes and ejection fraction, using previously validated commercially available software (CardIQ physio; GE Healthcare) (13). Reduced cardiac function was defined as a rest LV ejection fraction below $45 \%$ (14).

Quantification of MBF and MFR. Myocardial activity in the last frame of the dynamic datasets was volumetrically sampled, and polar maps of the LV were generated (15). Segments were applied to the whole series to obtain myocardial time-activity curves. A small region of interest was positioned in the LV cavity to obtain the arterial input function. MBF was then quantified by a retention approach. Myocardial activity concentration between minutes 4 and 8 was normalized to the area under the arterial input function in the first $120 \mathrm{~s}$. The resulting index was corrected for partial volume, spillover, and nonlinear extraction of ${ }^{82} \mathrm{Rb}(16)$. Validity and reproducibility of this approach were recently established for 
TABLE 1

Patient Profile

\begin{tabular}{|c|c|c|c|}
\hline Parameter & Control (eGFR $\geq 60$ ) & CKD (eGFR < 60) & $P$ \\
\hline Number (\%) & & & 0.48 \\
\hline Total & 44 & 36 & \\
\hline Male & 12 & 11 & \\
\hline Percentage & 38 & 39 & \\
\hline Age $(y)$ & $59.3 \pm 9.5$ & $62.6 \pm 12.0$ & 0.84 \\
\hline Body mass index & $36.5 \pm 9.5$ & $31.2 \pm 8.8$ & 0.08 \\
\hline \multicolumn{4}{|l|}{ Risk factor } \\
\hline Smoking & $10(11)$ & $7(8)$ & 0.20 \\
\hline Family history of CAD & $8(38)$ & $10(39)$ & 0.78 \\
\hline Hypertension & $24(55)$ & $23(61)$ & 0.90 \\
\hline Diabetes & $14(49)$ & $16(50)$ & 0.86 \\
\hline Dyslipidemia & $17(48)$ & $18(49)$ & 0.97 \\
\hline \multicolumn{4}{|l|}{ Medication at PET } \\
\hline Statin & $10(23)$ & $8(22)$ & 0.77 \\
\hline$\beta$-blocker & $16(36)$ & $10(27)$ & 0.36 \\
\hline Insulin & $2(38)$ & $7(17)$ & 0.15 \\
\hline Ace inhibitor or angiotensin receptor blocker & $15(34)$ & $18(50)$ & 0.22 \\
\hline \multicolumn{4}{|l|}{ Rest PET } \\
\hline Heart rate $(\mathrm{bpm})$ & $70.8 \pm 11.7$ & $75.0 \pm 16.9$ & 0.60 \\
\hline Systolic blood pressure (mm Hg) & $150.5 \pm 21.5$ & $150.7 \pm 24.4$ & 0.23 \\
\hline Diastolic blood pressure $(\mathrm{mm} \mathrm{Hg})$ & $73.9 \pm 11.9$ & $75.5 \pm 13.4$ & 0.92 \\
\hline Rate-pressure product (mm Hg/min) & $10,230.3 \pm 2,699.7$ & $11,313.1 \pm 3,343.0$ & 0.17 \\
\hline Ejection fraction (\%) & $55.5 \pm 10.1$ & $54.3 \pm 11.7$ & 0.80 \\
\hline \multicolumn{4}{|l|}{ Stress PET } \\
\hline Heart rate (bpm) & $89.7 \pm 13.8$ & $91.9 \pm 16.9$ & 0.41 \\
\hline Systolic blood pressure (mm Hg) & $153.1 \pm 26.1$ & $154.1 \pm 31.4$ & 0.24 \\
\hline Diastolic blood pressure $(\mathrm{mm} \mathrm{Hg})$ & $71.8 \pm 10.7$ & $72.9 \pm 13.6$ & 0.96 \\
\hline Rate-pressure product (mm Hg/min) & $13,775.2 \pm 3,465.8$ & $14,240.9 \pm 4,150.4$ & 0.46 \\
\hline Ejection fraction (\%) & $62.7 \pm 9.3$ & $62.6 \pm 11.2$ & 0.78 \\
\hline
\end{tabular}

Data are mean $\pm \mathrm{SD}$, or $n$ followed by percentage in parentheses.

our PET system (17). Rest MBF was normalized by dividing by the rate-pressure product and multiplying the result by 10,000 (18). MFR was determined as the ratio of stress to corrected rest MBF.

\section{Statistical Analysis}

Data are shown as mean \pm SD. Continuous variables were compared between 2 groups using the unpaired Student $t$ test. Categoric variables were compared using the Fisher exact test. The Pearson correlation coefficient was used to describe the relationship between continuous variables. Multivariate regression analysis was performed using stepwise forward logistic regression. Reported $P$ values are 2 -sided, and less than 0.05 was considered statistically significant. The statistical analysis was performed using SPSS (version 11; IBM SPSS).

\section{RESULTS}

\section{Clinical Parameters}

Table 2 summarizes available laboratory results. As expected, serum creatinine was significantly higher in CKD than in controls $(1.9 \pm 1.1$ vs. $0.8 \pm 0.2 \mathrm{mg} / \mathrm{dL}, P<$ $0.001)$. Both hemoglobin and hematocrit were significantly lower in CKD (hemoglobin, $13.1 \pm 4.8$ vs. $11.3 \pm 5.6$ $\mathrm{g} / \mathrm{dL}, P=0.02$; hematocrit, $38.9 \% \pm 3.9 \%$ vs. $36.1 \% \pm$ $5.4 \%$ in control group and CKD, $P=0.014$ ). And pro-B- type natriuretic peptide was significantly higher in CKD than in controls $(P=0.005)$.

Lipid and glucose levels, as well as the coronary calcium score according to Agatston, obtained by a prospectively gated CT scan during the PET/CT session, were not different between groups.

\section{Global MBF and MFR}

Significantly lower MFR was observed in CKD than in control patients $(2.2 \pm 1.0$ vs. $3.0 \pm 1.2, P=0.027)$. Notably, stress MBF was comparable between groups (2.3 \pm 0.9 vs. $2.3 \pm 0.8 \mathrm{~mL} / \mathrm{min} / \mathrm{g}, P=0.08$ ) (Fig. 2 ), but rest $\mathrm{MBF}$ was significantly increased in $\mathrm{CKD}$, both before and after correction for baseline work $(1.2 \pm 0.6$ vs. $0.8 \pm 0.2 \mathrm{~mL} / \mathrm{min} / \mathrm{g}, P=0.001$, before correction; $1.1 \pm 0.4$ vs. $0.8 \pm 0.1 \mathrm{~mL} / \mathrm{min} / \mathrm{g}, P=0.007$, after correction) (Fig. 3).

\section{Correlation Between Flow and eGFR}

When all 82 patients of both groups were combined, MFR showed a significant positive correlation with eGFR ( $r=0.46 ; P<0.0001)$, whereas stress MBF did not $(r=$ $0.002 ; P=0.45)$. Consistent with group differences, rest MBF showed a significant inverse correlation with eGFR 
TABLE 2

Clinical Pathology

\begin{tabular}{|c|c|c|c|c|c|}
\hline \multirow[b]{2}{*}{ Variable } & \multicolumn{2}{|c|}{ Control (eGFR $\geq 60$ ) } & \multicolumn{2}{|c|}{ CKD (eGFR < 60) } & \multirow[b]{2}{*}{$P$} \\
\hline & Mean $\pm S D$ & $n$ & Mean $\pm S D$ & $n$ & \\
\hline eGFR $\left(\mathrm{mL} / \mathrm{min} / 1.73 \mathrm{~m}^{2}\right)$ & $99.2 \pm 28.2$ & 42 & $43.7 \pm 13.8$ & 40 & $<0.0001$ \\
\hline Serum creatinine (mg/dL) & $0.8 \pm 0.2$ & 42 & $1.9 \pm 1.1$ & 40 & $<0.0001$ \\
\hline Hemoglobin (g/dL) & $13.1 \pm 4.8$ & 39 & $11.3 \pm 5.6$ & 33 & 0.02 \\
\hline Hematocrit (\%) & $38.9 \pm 3.9$ & 39 & $36.1 \pm 5.4$ & 33 & 0.014 \\
\hline Glucose (mg/dL) & $124.6 \pm 51.8$ & 39 & $124.6 \pm 51.8$ & 36 & 0.43 \\
\hline HbA1c (for diabetic patients) (\%) & $8.4 \pm 2.5$ & 17 & $7.3 \pm 1.9$ & 14 & 0.35 \\
\hline Total cholesterol (mg/dL) & $174.6 \pm 38.1$ & 19 & $180.0 \pm 35.8$ & 17 & 0.32 \\
\hline Low-density lipoprotein cholesterol (mg/dL) & $100.7 \pm 36.5$ & 19 & $96.9 \pm 19.5$ & 17 & 0.77 \\
\hline Triglycerides $(\mathrm{mg} / \mathrm{dL})$ & $140.5 \pm 116.4$ & 19 & $120.1 \pm 52.6$ & 17 & 0.87 \\
\hline High-density lipoprotein cholesterol (mg/dL) & $51.9 \pm 16.7$ & 19 & $54.6 \pm 19.5$ & 17 & 0.83 \\
\hline Pro-B-type natriuretic peptide (pg/dL) & $149.4 \pm 154.4$ & 11 & $971 \pm 1112$ & 12 & 0.005 \\
\hline Agatston score & $125.0 \pm 448.6$ & 42 & $284.0 \pm 559.9$ & 40 & 0.24 \\
\hline
\end{tabular}

both before $(r=-0.44 ; P=0.0003)$ and after $(r=-0.49$; $P<0.0001)$ correction for baseline cardiac work (Fig. 4).

Also, hemoglobin showed a significant inverse correlation with rest $\mathrm{MBF}(r=-0.28 ; P=0.014)$. To determine independent correlates of rest $\mathrm{MBF}$, multiple logistic regression analysis was performed by forming equal groups of high or low rest MBF using the median value $(0.94 \mathrm{~mL} /$ $\mathrm{min} / \mathrm{g}$ ) and including eGFR, creatinine, and hemoglobin in the model. In this multivariate analysis, only eGFR was an independent predictor of increased rest MBF; hemoglobin and creatinine were not (Table 3 ).

\section{DISCUSSION}

Our study shows that global MFR is reduced in patients with CKD and an otherwise normal clinical myocardial perfusion PET scan result. The reduction of flow reserve was mostly attributed to an elevated baseline flow, which was independently associated with eGFR, supporting the notion of microvascular dysfunction in this patient group. Our study confirms a link between renal function and the coronary microcirculation. It also highlights a strength of quantitative PET, which is capable of detecting global abnormalities of the microcirculation, even when regional ischemia as a marker of macroscopic flow-limiting disease is absent.
Renal dysfunction is known to be a coronary risk factor that is associated with coronary atherosclerosis. Epidemiologic studies have shown that cardiac and renal failure have synergistic effects regarding adverse outcome (19). Al-Mallah et al., using standard myocardial perfusion SPECT, reported in a large population that the frequency of regional perfusion abnormalities was almost doubled in patients with CKD, compared with those without, whereas an abnormal scan result retained its incremental value to predict adverse outcome (20). Similar findings were observed in less advanced renal dysfunction (21).

Our study focuses on subjects with CKD but without overt ischemic heart disease. None of the patients in our CKD group had relative regional perfusion abnormalities or depressed LV function, and the degree of reduction of eGFR and creatinine was mild, suggesting that our patients represent a group with early disease, in which macroscopic flow-limiting changes of the major coronary arteries are not yet present. Even in this setting, the coronary circulation seems to be impaired. Endothelial dysfunction may be a major contributor to our observation of reduced flow reserve. It is thought to reflect impaired regulation of vascular tone as an occult, early stage in the development of atherosclerosis, before the occurrence of regional perfusion deficits. This concept of microvascular dysfunction

FIGURE 2. Comparison of MFR and stress flow for CKD and matched control group (Cont). MFR was significantly lower in CKD than in controls (A). Both groups were comparable for stress MBF (B).

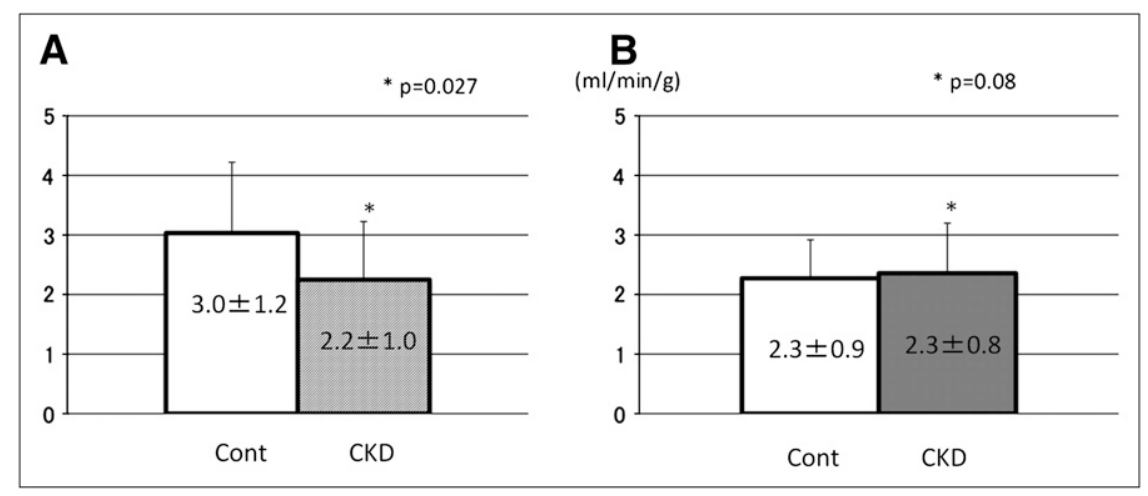




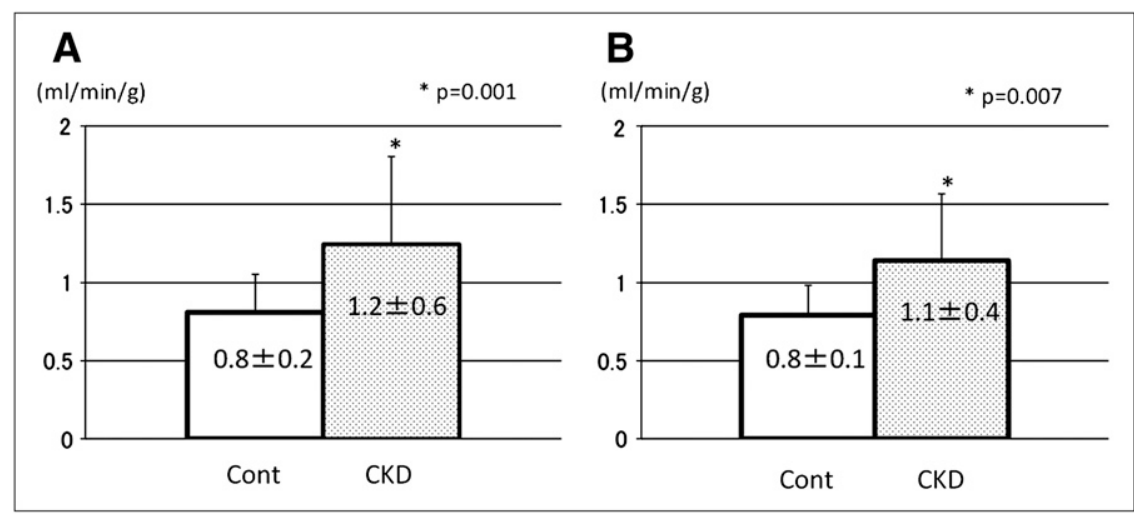

FIGURE 3. Comparison of rest MBF for CKD and matched control group (Cont). There was significant difference between groups for rest flow before $(A)$ and after $(B)$ correction for cardiac work. as a precursor of clinical coronary disease has been tested in multiple prior studies in well-selected subject groups with individual cardiovascular risk factors $(18,22,23)$ and in response to modification of these risk factors $(23,24)$. More recently, the evidence that impairments of global flow reserve have a prognostic value for subsequent progression toward overt coronary disease or cardiovascular events is increasing $(7,8,25,26)$. Hence, although not tested directly in this study, it is conceivable that impaired global flow reserve in our CKD subjects reflects a state of elevated risk.

Interestingly, reduced MFR was mainly due to significantly increased rest flow in our CKD patients and not to attenuated stress flow. This association may point toward a state of baseline endothelial activation that has previously been observed in other conditions such as diabetes mellitus (27) or hemodialysis patients $(28,29)$.

There have been few reports on the characteristics of myocardial flow in CKD using nuclear imaging techniques. Koivuviita et al., using ${ }^{15} \mathrm{O}$-water, also reported that base- line myocardial flow was elevated in concordance with the severity of renal dysfunction whereas flow reserve was preserved. Their study enrolled patients with various stages of CKD but at relatively small numbers. No specific exclusion of subjects with regional perfusion abnormality was performed, although the contribution of macroscopic disease and ischemia was probably low because of lack of decreased stress flow (30).

The exact mechanisms by which coronary microcirculation is impaired in renal dysfunction cannot be elucidated from this study. Neurohumoral activation, which is present in CKD, may be one contributor (31). Also, anemia is a dominant feature in CKD, and it leads to a hyperdynamic state and to vasodilation via nitric oxide release to improve peripheral hypoxia (32) - possibly another contributor to increased rest flow in renal dysfunction. Although some investigators reported that nitric oxide synthesis might be reduced, others reported that nitric oxide was increasingly released in patients with renal disease (33) and appeared to

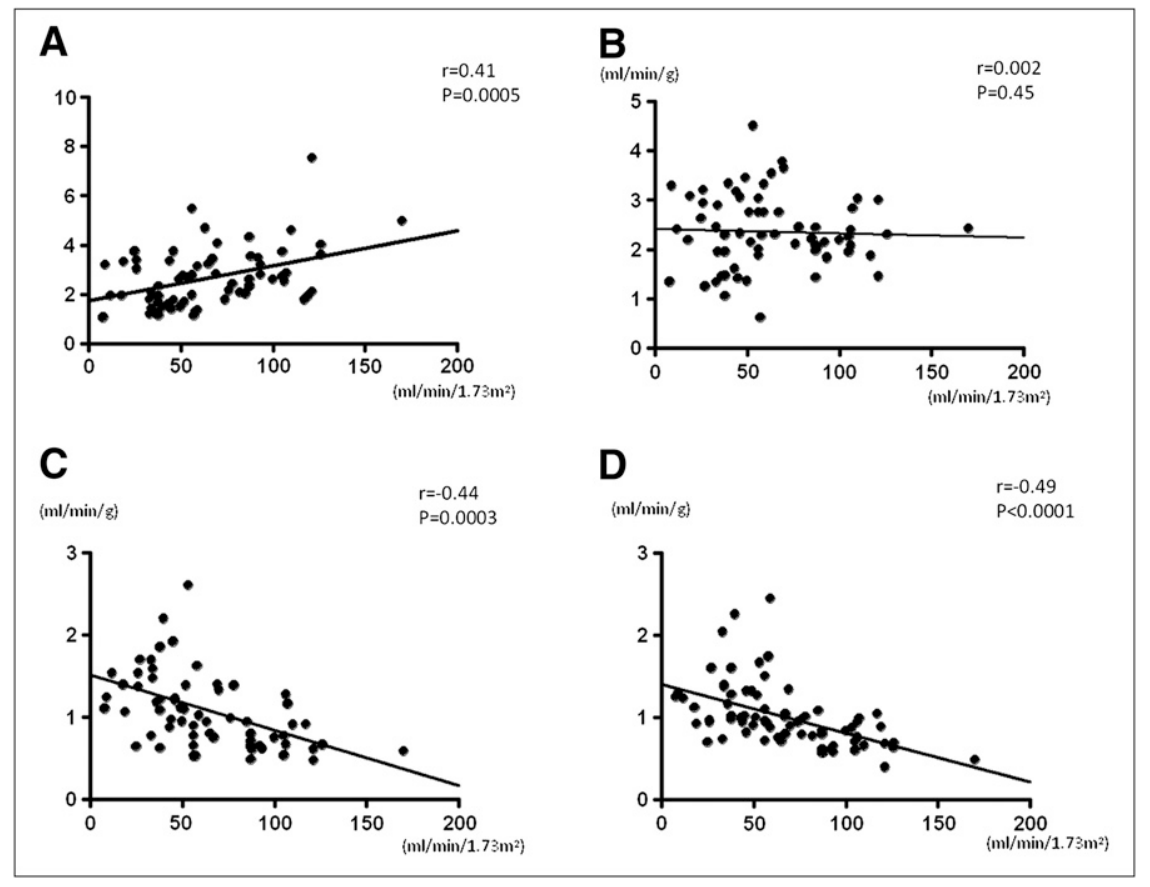

FIGURE 4. Regression plots for eGFR and MFR (A), stress MBF (B), and rest MBF before $(C)$ and after $(D)$ correction for cardiac work. 
TABLE 3

Multiple Logistic Regression Model Estimates for Rest Myocardial Flow Above Median

\begin{tabular}{lccl}
\hline \multicolumn{1}{c}{ Variable } & $\begin{array}{c}\text { Odds } \\
\text { ratio }\end{array}$ & $\begin{array}{c}95 \% \text { confidence } \\
\text { interval }\end{array}$ & $P$ \\
\hline eGFR $\left(\mathrm{mL} / \mathrm{min} / 1.73 \mathrm{~m}^{2}\right)$ & 5.61 & $1.16-12.98$ & 0.003 \\
Creatinine $(\mathrm{mg} / \mathrm{dL})$ & 3.44 & $1.02-7.56$ & 0.07 \\
Hemoglobin $(\mathrm{g} / \mathrm{dL})$ & 2.50 & $0.72-5.31$ & 0.81 \\
\hline
\end{tabular}

be mediated by angiotensin II in endothelial cells (34). Increased rest flow is probably reflecting a state of endothelial activation in our study. Anemia, which is a frequent finding in CKD, may be a contributor to increased rest flow, but our multivariate analysis, in which hemoglobin was not an independent predictor of elevated rest flow, suggests that it is not a dominant factor.

Our study has some limitations. First, it is a retrospective analysis with all its inherent limitations. Matching of subjects with and without CKD was done carefully, but the lack of prospective inclusion criteria may limit statistical power. Also, additional risk parameters (35) are missing, or the included clinical or pathologic markers were incomplete. Because of a thorough selection, sample size was small, but results were conclusive. The small sample size did not allow for outcome analysis, but our study should be seen as a hypothesis generator for future, larger prospective registries to identify a potential prognostic value of decreased flow reserve in CKD. Second, no information about coronary anatomy was available. The absence of relative perfusion abnormalities may not fully exclude severe and balanced ischemia. But the elevation of rest flow as the major determinant of impaired flow reserve in our study cannot be explained by flow-limiting coronary disease. And finally, our analysis was done with subjects referred for a clinical myocardial perfusion study. Hence, even the subjects without CKD exhibited risk factors and clinical characteristics that led to the decision of ordering the scan. The degree of abnormality may be underestimated when using such a group for comparison. Nevertheless, our analysis allowed for detection of significant differences in a conclusive manner.

\section{CONCLUSION}

In early-stage CKD, in the absence of regional ischemia or impaired LV function, impaired MFR due to increased rest MBF is detectable by quantitative ${ }^{82} \mathrm{Rb}$ myocardial perfusion PET. This may be a hallmark of endothelial activation, which may contribute to accelerated progression toward overt coronary artery disease.

\section{DISCLOSURE STATEMENT}

The costs of publication of this article were defrayed in part by the payment of page charges. Therefore, and solely to indicate this fact, this article is hereby marked "advertisement" in accordance with 18 USC section 1734.

\section{ACKNOWLEDGMENTS}

This study was supported in part by a Wagner-Torizuka Fellowship grant of the SNM and by GE Healthcare, Bracco Diagnostics, and Lantheus Medical Imaging. Frank Bengel has served as a consultant to Lantheus Medical Imaging, Astellas Pharma, and GE Healthcare. No other potential conflict of interest relevant to this article was reported.

\section{REFERENCES}

1. Bock JS, Gottlieb SS. Cardiorenal syndrome: new perspectives. Circulation. 2010;121:2592-2600.

2. Shlipak MG, Massie BM. The clinical challenge of cardiorenal syndrome. Circulation. 2004;110:1514-1517.

3. Bleyer AJ, Hartman J, Brannon PC, Reeves-Daniel A, Satko SG, Russell G. Characteristics of sudden death in hemodialysis patients. Kidney Int. 2006;69:22682273.

4. Dries DL, Exner DV, Domanski MJ, Greenberg B, Stevenson LW. The prognostic implications of renal insufficiency in asymptomatic and symptomatic patients with left ventricular systolic dysfunction. J Am Coll Cardiol. 2000;35:681-689.

5. Camici PG, Crea F. Coronary microvascular dysfunction. N Engl J Med. 2007; 356:830-840.

6. Bengel FM. Leaving relativity behind: quantitative clinical perfusion imaging. J Am Coll Cardiol. 2011;58:749-751.

7. Fukushima K, Javadi MS, Higuchi T, et al. Prediction of short-term cardiovascular events using quantification of global myocardial flow reserve in patients referred for clinical ${ }^{82} \mathrm{Rb}$ PET perfusion imaging. J Nucl Med. 2011;52:726-732.

8. Ziadi MC, Dekemp RA, Williams KA, et al. Impaired myocardial flow reserve on rubidium- 82 positron emission tomography imaging predicts adverse outcomes in patients assessed for myocardial ischemia. J Am Coll Cardiol. 2011;58:740-748.

9. Levey AS, Bosch JP, Lewis JB, Greene T, Rogers N, Roth D. A more accurate method to estimate glomerular filtration rate from serum creatinine: a new prediction equation. Modification of Diet in Renal Disease Study Group. Ann Intern Med. 1999;130:461-470.

10. Lautamäki R, Brown TL, Merrill J, Bengel FM. CT-based attenuation correction in (82)Rb-myocardial perfusion PET-CT: incidence of misalignment and effect on regional tracer distribution. Eur J Nucl Med Mol Imaging. 2008;35:305-310.

11. Cerqueira MD, Weissman NJ, Dilsizian V, et al. Standardized myocardial segmentation and nomenclature for tomographic imaging of the heart: a statement for healthcare professionals from the Cardiac Imaging Committee of the Council on Clinical Cardiology of the American Heart Association. Circulation. 2002;105:539-542.

12. Hansen CL, Goldstein RA, Akinboboye OO, et al. Myocardial perfusion and function: single photon emission computed tomography. J Nucl Cardiol. 2007; 14:e39-e60.

13. Chander A, Brenner M, Lautamaki R, Voicu C, Merrill J, Bengel FM. Comparison of measures of left ventricular function from electrocardiographically gated ${ }^{82} \mathrm{Rb}$ PET with contrast-enhanced CT ventriculography: a hybrid PET/CT analysis. J Nucl Med. 2008;49:1643-1650.

14. Bravo PE, Chien D, Javadi M, Merrill J, Bengel FM. Reference ranges for LVEF and LV volumes from electrocardiographically gated ${ }^{82} \mathrm{Rb}$ cardiac PET/CT using commercially available software. J Nucl Med. 2010;51:898-905.

15. Nekolla SG, Miethaner C, Nguyen N, Ziegler SI, Schwaiger M. Reproducibility of polar map generation and assessment of defect severity and extent assessment in myocardial perfusion imaging using positron emission tomography. Eur $J$ Nucl Med. 1998;25:1313-1321.

16. Yoshida K, Mullani N, Gould KL. Coronary flow and flow reserve by PET simplified for clinical applications using rubidium-82 or nitrogen-13-ammonia. J Nucl Med. 1996;37:1701-1712.

17. Lautamäki R, George RT, Kitagawa K, et al. Rubidium-82 PET-CT for quantitative assessment of myocardial blood flow: validation in a canine model of coronary artery stenosis. Eur J Nucl Med Mol Imaging. 2009;36:576-586.

18. Czernin J, Muller P, Chan S, et al. Influence of age and hemodynamics on myocardial blood flow and flow reserve. Circulation. 1993;88:62-69.

19. Go AS, Chertow GM, Fan D, McCulloch CE, Hsu CY. Chronic kidney disease and the risks of death, cardiovascular events, and hospitalization. $N$ Engl J Med. 2004;351:1296-1305.

20. Al-Mallah MH, Hachamovitch R, Dorbala S, Di Carli MF. Incremental prognostic value of myocardial perfusion imaging in patients referred to stress single- 
photon emission computed tomography with renal dysfunction. Circ Cardiovasc Imaging. 2009;2:429-436.

21. Coceani M, Gimelli A, Carpeggiani C, L'Abbate A, Marzullo P. Clinical utility of estimated glomerular filtration rate in patients undergoing gated SPECT. J Nucl Cardiol. 2009; 16:384-390.

22. Czernin J, Barnard RJ, Sun KT, et al. Effect of short-term cardiovascular conditioning and low-fat diet on myocardial blood flow and flow reserve. Circulation. 1995;92:197-204.

23. Kaufmann PA, Gnecchi-Ruscone T, di Terlizzi M, Schafers KP, Luscher TF, Camici PG. Coronary heart disease in smokers: vitamin $\mathrm{C}$ restores coronary microcirculatory function. Circulation. 2000;102:1233-1238.

24. Higuchi T, Abletshauser C, Nekolla SG, Schwaiger M, Bengel FM. Effect of the angiotensin receptor blocker Valsartan on coronary microvascular flow reserve in moderately hypertensive patients with stable coronary artery disease. Microcirculation. 2007;14:805-812.

25. Britten MB, Zeiher AM, Schachinger V. Microvascular dysfunction in angiographically normal or mildly diseased coronary arteries predicts adverse cardiovascular long-term outcome. Coron Artery Dis. 2004;15:259-264.

26. Herzog BA, Husmann L, Valenta I, et al. Long-term prognostic value of ${ }^{13} \mathrm{~N}$-ammonia myocardial perfusion positron emission tomography added value of coronary flow reserve. J Am Coll Cardiol. 2009;54:150-156.

27. Ragosta M, Samady H, Isaacs RB, Gimple LW, Sarembock IJ, Powers ER. Coronary flow reserve abnormalities in patients with diabetes mellitus who have end-stage renal disease and normal epicardial coronary arteries. Am Heart J. 2004;147:1017-1023.

28. Niizuma S, Takiuchi S, Okada S, et al. Decreased coronary flow reserve in haemodialysis patients. Nephrol Dial Transplant. 2008;23:2324-2328.

29. Tok D, Gullu H, Erdogan D, et al. Impaired coronary flow reserve in hemodialysis patients: a transthoracic Doppler echocardiographic study. Nephron Clin Pract. 2005;101:c200-c206.

30. Koivuviita N, Tertti R, Jarvisalo M, et al. Increased basal myocardial perfusion in patients with chronic kidney disease without symptomatic coronary artery disease. Nephrol Dial Transplant. 2009;24:2773-2779.

31. Kurata C, Wakabayashi Y, Shouda S, et al. Enhanced cardiac clearance of iodine123-MIBG in chronic renal failure. J Nucl Med. 1995;36:2037-2043.

32. Crawford JH, Isbell TS, Huang Z, et al. Hypoxia, red blood cells, and nitrite regulate NO-dependent hypoxic vasodilation. Blood. 2006;107:566-574.

33. Roccatello D, Mengozzi G, Alfieri V, et al. Early increase in blood nitric oxide, detected by electron paramagnetic resonance as nitrosylhaemoglobin, in haemodialysis. Nephrol Dial Transplant. 1997;12:292-297.

34. Patzak A, Persson AE. Angiotensin II-nitric oxide interaction in the kidney. Curr Opin Nephrol Hypertens. 2007;16:46-51.

35. Stevens LA, Li S, Kurella Tamura M, et al. Comparison of the CKD Epidemiology Collaboration (CKD-EPI) and Modification of Diet in Renal Disease (MDRD) study equations: risk factors for and complications of CKD and mortality in the Kidney Early Evaluation Program (KEEP). Am J Kidney Dis. 2011; 57(3, suppl 2):S9-S16. 